\section{BIOMARCADORES Y MEDICINA PERSONALIZADA EN LAS ENFERMEDADES RARAS}

\author{
Francesc Palau \\ Hospital Sant Joan de Déu \\ CIBER de Enfermedades Raras - CIBERER \\ Instituto de Salud Carlos III \\ Institut Clínic de Medicina i Dermatologia (ICMiD) \\ Hospital Clínic, Barcelona \\ Universitat de Barcelona \\ ORCID iD: https://orcid.org/0000-0002-8635-5421 \\ fpalau@sjdhospitalbarcelona.org
}

Cómo citar este artículo/Citation: Palau, F. (2018). Biomarcadores y medicina personalizada en las enfermedades raras. Arbor, 194 (789): a465. https://doi.org/10.3989/ arbor.2018.789n3007

Recibido: 30 abril 2015. Aceptado: 13 mayo 2016.

RESUMEN: Las enfermedades raras, más allá de su baja prevalencia, presentan diferencias con las enfermedades comunes. Los biomarcadores se caracterizan por ser herramientas útiles para determinar y evaluar de un modo objetivo procesos biológicos normales, procesos patológicos o la respuesta farmacológica a las intervenciones terapéuticas o a cualquier otra intervención en la atención sanitaria. Como determinantes biológicos de la enfermedad humana y del modo de enfermar individual, los biomarcadores son de especial interés para el estudio de las enfermedades y el seguimiento de las respuestas terapéuticas de los nuevos medicamentos. En el ámbito de las enfermedades raras, los biomarcadores genéticos y genómicos permiten realizar el diagnóstico de la mutación primaria y determinar el perfil genómico pero, al igual que ocurre con las enfermedades comunes complejas, los biomarcadores bioquímicos y los perfiles ómicos dinámicos y funcionales son fundamentales para el diseño de la medicina personalizada en los pacientes con enfermedades poco frecuentes.

PALABRAS CLAVE: Biomarcadores; enfermedades raras; medicina personalizada; genómica; proteómica; metabolómica.

\section{BIOMARKERS AND PERSONALIZED MEDICINE IN RARE DISEASES}

Copyright: (c) 2018 CSIC. Este es un artículo de acceso abierto distribuido bajo los términos de la licencia de uso y distribución Creative Commons Reconocimiento 4.0 Internacional (CC BY 4.0).
ABSTRACT: Rare diseases differ from common diseases, beyond their low prevalence. Biomarkers are characterized as useful tools to objectively identify and evaluate normal biological processes, pathological processes, or the pharmacological response to therapeutic or other healthcare interventions. As biological determinants of human disease biomarkers are of special interest for the study of diseases and to monitor the therapeutic responses of new drugs. In the field of rare diseases, genetic and genomic biomarkers are essential to determine the primary mutation and genomic profile but, as with common complex diseases, both biochemical biomarkers and the dynamic and functional omic profiles are fundamental to design the personalized medicine for patients with rare diseases.

KEYWORDS: Biomarkers; rare diseases; personalized medicine; genomics; proteomics; metabolomics. 


\section{INTRODUCCIÓN}

La definición de medicina personalizada no es una cuestión fácil (Ginsburg, 2009; Ginsburg y Willard, 2010; Hamburg y Collins, 2010). En el contexto de la atención sanitaria orientada al individuo se viene proponiendo en los últimos años la transición desde una práctica médica personalizada al concepto moderno de la personalización de la ciencia médica para mejorar la salud individual y el tratamiento individualizado para cada paciente. En muchos aspectos la medicina personalizada, estratificada o de precisión se refiere a la incorporación de la individualidad biológica única del individuo y su interacción con el medio ambiente en la medicina clínica y cuidado de la salud de la persona. Por medicina personalizada entendemos la integración de la información que se proporciona a una persona en particular y el uso en la práctica clínica de la interacción entre el fenotipo y los perfiles genético, genómico, transcriptómico, proteómico y metabolómico del individuo con el medio ambiente, incluyendo el entorno nutricional (Palau, 2012). La consecuencia esperada es optimizar las estrategias de salud preventiva y de respuesta a las terapias con medicamentos, actuando de un modo anticipatorio mientras las personas están aún en un estado de salud o en una etapa temprana de la enfermedad. La medicina personalizada trata de trasladar la evidencia de las intervenciones médicas basada en el conocimiento de la población como conjunto de individuos hacia la evidencia individual de cómo tratar a la persona específica basada en su historia clínica, su perfil biológico y su hábitat natural. El objetivo es ofrecer una asistencia sanitaria adaptada a cada persona. La medicina personalizada, fundamentada en la integración de la información individual, a partir de la variación del genoma, la fisiología y el fenotipo celular en su interacción con el entorno personal, puede representar un modelo proactivo, preventivo y prospectivo de atención sanitaria (Snyderman, 2009; Snyderman y Langheier, 2006; Snyderman y Yoediono, 2006), por oposición a aquella que pivota alrededor de la enfermedad con un enfoque tradicional, reactivo, del estado de salud de los individuos. Este enfoque de la medicina personalizada afecta tanto a las enfermedades comunes como a las enfermedades raras.

\section{ENFERMEDADES RARAS Y SU RELACIÓN CON LAS ENFERMEDADES COMUNES}

La investigación en enfermedades raras (ER) ha devenido en un elemento esencial para comprender las causas y mecanismos de la enfermedad humana y de los modos de enfermar en las personas. En las pasadas tres décadas ha habido un importante y creciente avance en el diagnóstico de las enfermedades raras y, en menor medida, en el tratamiento de las mismas. Por el contrario, durante este mismo período ha habido un relativo estancamiento en el progreso médico y científico de las enfermedades comunes. El conocimiento generado por la genética en el ámbito de las enfermedades minoritarias y de la biología de nuevas rutas celulares y moleculares relacionadas con las mismas está contribuyendo también a conocer mejor la patogénesis tanto de las propias enfermedades raras como de las enfermedades comunes (Roubertoux y de Vries, 2011).

Se tiende a pensar que las enfermedades raras son -aparentemente- más simples que las enfermedades frecuentes. En la mayoría de las enfermedades raras la mutación en un gen es condición necesaria, y en muchas ocasiones suficiente, para que la enfermedad se manifieste en algún momento de la vida. Así pues, podríamos caer en la tentación de pensar que la enfermedad rara se puede concebir como un experimento controlado de la naturaleza en la que todo es idéntico en la persona enferma y en las personas sanas, excepto por un solo factor que es la causa fundamental de la enfermedad, la mutación génica. Nada más lejos de la realidad: la fisiopatología de las enfermedades raras y sus variantes fenotípicas son la consecuencia de alteraciones en redes biológicas complejas, que condicionan formas complejas de enfermar en las personas afectadas por una de estas enfermedades poco frecuentes. Las mismas vías biológicas 'simples' que se encuentran en las enfermedades raras son también componentes de la fisiopatología de las enfermedades comunes. Si en términos de prevalencia y carga asistencial las enfermedades comunes son rompecabezas que los investigadores médicos han de resolver, las enfermedades raras son piezas fundamentales de tales rompecabezas, al tiempo que un problema global de salud pública. No es descabellado proponer que las enfermedades raras constituyen el extremo de la expresión patológica y clínica, en forma de fenotipos específicos, del continuum de la vida humana y de las enfermedades comunes.

Las enfermedades raras, no obstante, se reconocen como enfermedades poco frecuentes, con una prevalencia de menos de cinco afectados por 10.000 habitantes y con una serie de características condicionadas por fenotipos clínicos generalmente crónicos e invalidantes. Conceptualmente podemos considerar que los elementos que conforman el marco de las en- 
fermedades raras son aún más amplios y que se pueden resumir del modo que sigue (Palau, 2010):

Componente de salud pública. Cada enfermedad rara es muy poco prevalente; sin embargo, la afectación grosso modo de 3.000 .000 de personas con problemas de salud compartidos -y, claro está, con otros muchos no compartidos- en una población como la española tomada en su globalidad, sí es una cuestión que requiere una aproximación sanitaria y científica desde los supuestos de la salud pública. Es lo que se ha denominado como la paradoja de la rareza.

Historia natural: diversidad y heterogeneidad. En un conjunto de afecciones tan amplio como el de las enfermedades raras la diversidad es una característica intrínseca. La naturaleza de los procesos patológicos varía desde enfermedades que afectan a un único sistema orgánico hasta enfermedades que son multisistémicas. La heterogeneidad de estas enfermedades se manifiesta en distintos perfiles de su historia natural, lo cual condiciona la actuación clínica y preventiva de los servicios de salud.

Atención integral y multidisciplinar. Una enfermedad rara suele ser crónica e incapacitante. En muchas ocasiones el paciente con este tipo de enfermedades necesita una atención que vaya más allá de la asistencia clínica específica que se le ofrece desde un servicio especializado. Esta atención se debe plantear en el contexto del manejo global, con atención médica integral, psicológica y social.

Componente socio-sanitario. Muchas enfermedades raras suponen un alto grado de dependencia y de carga social, sanitaria y económica. Esto no es algo particular de las enfermedades raras, pero hay aspectos que sí son específicos de las mismas y que les confieren cierto grado de particularidad.

Herencia genética. Se estima que cuatro quintas partes de las enfermedades raras son genéticas, mayoritariamente monogénicas, y siguen las leyes de la herencia mendeliana.

Riesgo de recurrencia de una enfermedad genética en las familias, lo que hace del consejo genético una herramienta terapéutica de primer orden para estos pacientes y sus familiares.

Dispersión geográfica. La distribución de los enfermos y de las familias por una geografía amplia es un fenómeno intrínseco a las enfermedades raras. La distribución aleatoria y la baja prevalencia hacen que pueda haber casos en cualquier lugar, sea un medio urbano o rural, sin que se concentren en ningún área concreta.

Oportunidades de tratamiento y desarrollo de terapias. Las opciones terapéuticas en la enfermedad rara son, en general, escasas y poco eficaces. No obstante, el desarrollo de nuevas terapias y fármacos para estos trastornos dirigidos a dianas moleculares específicas de enfermedades raras puede ser no solo útil para las mismas sino también para enfermedades comunes que puedan compartir tales dianas biológicas.

Teniendo estos elementos en cuenta, junto con la prevalencia, podemos hacer una distinción entre las enfermedades raras y las enfermedades comunes, para determinar qué las diferencia y hace que sea relevante en términos médicos y de salud pública reconocer al amplio y variado grupo de las enfermedades raras (Berman, 2014):

Las enfermedades raras se manifiestan típicamente y con mayor frecuencia en la población joven, esto es, en la época pediátrica, considerada esta como la lactancia, la infancia y la adolescencia, a lo largo de las dos primeras décadas de la vida, cuando el individuo crece y se desarrolla física y mentalmente. También aparecen en la edad adulta, pero en este período de la vida son más frecuentes las enfermedades comunes.

Las enfermedades raras suelen seguir un patrón genético, con herencia mendeliana o mitocondrial. Las enfermedades comunes pueden darse en familias pero generalmente no siguen un patrón mendeliano.

En ambos casos influye el componente epigenético y la interacción con el medio ambiente, pero esto es más intenso y juegan un papel mayor en la patogenia de las enfermedades comunes.

Las enfermedades raras se manifiestan a menudo como cuadros sindrómicos, afectando varios órganos o sistemas fisiológicos, con participación de rutas metabólicas cuyo conocimiento va en aumento; las enfermedades comunes suelen ser no sindrómicas, aunque pueden compartir las mencionadas rutas biológicas, siendo este un punto de confluencia de primer orden para determinar nuevas dianas terapéuticas.

Las diferencias en la tasa de aparición de las enfermedades raras comparada con la de las enfermedades comunes es muy grande, siendo del orden de mil veces más o incluso mayor.

Existen muchas más enfermedades raras que enfermedades comunes. No obstante, está por establecer qué vías y redes biológicas y fisiopatológicas comparten.

\section{BIOMARCADORES}

El concepto de biomarcador se relaciona con la medición de determinantes y procesos bioquímicos, celulares o fisiológicos en cualquier material biológi- 
co. Centrándonos más en el plano de la enfermedad humana, los biomarcadores se caracterizan por ser herramientas para determinar y evaluar de un modo objetivo procesos biológicos normales, procesos patológicos, o la respuesta farmacológica de intervenciones terapéuticas o cualquier otra intervención en cuidados de la salud. Los biomarcadores se pueden aplicar como herramientas diagnósticas, de estadiaje y pronóstico de la enfermedad, y predicción y monitorización de la respuesta a un tratamiento farmacológico, a una terapia no farmacológica o a una intervención terapéutica de cualquier índole. La determinación de un biomarcador se puede hacer directamente en fluidos o tejidos corporales, o alternativamente mediante técnicas de imagen que pueden incluir trazadores específicos de funciones fisiológicas.

En el campo del descubrimiento y la aplicación clínica de los biomarcadores conviene tener en cuenta dos conceptos fundamentales: el objetivo subrogado (surrogate endpoint) y la validación (Bonassi y Neri, 2009). Un objetivo o producto subrogado es una medida de laboratorio o signo físico empleado en un ensayo clínico como sustituto de producto final medido directamente en el paciente, tal como las funciones vitales, la apreciación del paciente respecto a su situación clínica o la supervivencia. Por ejemplo, es esperable que los cambios inducidos por una terapia en un producto subrogado también se reflejen en un cambio significativo del resultado final clínico. El uso de un biomarcador como producto subrogado requiere su validación, esto es, la demostración de la precisión (correlación entre lo medido con el resultado clínico) y la reproductibilidad de lo que se está midiendo. El biomarcador subrogado debe ser un correlato del verdadero alcance clínico del procedimiento y capturar en su totalidad el efecto neto de un tratamiento en el resultado clínico. Siguiendo las pautas de las autoridades regulatorias, como es la Agencia Europea del Medicamento (EMA en sus siglas inglesas), las propiedades que un biomarcador ideal debería poseer son: (i) medir un parámetro específico en un rango que distinga entre el estatus patológico y el normal (validez analítica); (ii) los cambios observados en su determinación deben indicar un cambio en las características de la enfermedad con la que se correlaciona y los cambios de tales características de la enfermedad deben reflejarse en modificaciones en el propio biomarcador (validez clínica); (iii) los cambios medidos en el biomarcador reflejen con una alta probabilidad que se deben a un efecto de una intervención dada (utilidad clínica); (iv) un biomarcador debe ser fácil de identificar y de medir y no sufrir variación en función del tipo de recogida de las muestras, métodos y plataformas empleadas para su identificación (factibilidad); (v) tener en cuenta la relación coste-efectividad, que en la enfermedades de baja prevalencia también es importante por el coste elevado de cada tratamiento individual, y (vi) su determinación no debe requerir procedimientos invasivos (revisado en Ferlini, Scotton y Novelli, 2013; Scotton, Passarelli, Neri y Ferlini, 2014).

Por lo que respecta a los tipos de biomarcadores hay dos criterios para definirlos, relacionados bien con el momento del proceso clínico en que se aplican, bien en con el tipo de molécula biológica que se investiga o analiza (Ferlini et al., 2013; Scotton et al. 2014).

\section{Biomarcadores de proceso clínico}

En relación con el momento de la historia natural de la enfermedad cabe indicar que hay biomarcadores de exposición ambiental y biomarcadores de susceptibilidad, concretamente aquellos que infieren predisposición a desarrollar o proteger de la enfermedad según el perfil genético del individuo (Bonassi y Neri, 2009). En el caso de las enfermedades raras monogénicas la presencia de una mutación génica es indicativa de predisposición o diagnóstico de la enfermedad en cuestión. En este contexto cabe distinguir dos tipos de biomarcadores según su ubicación en la historia natural: (i) biomarcador diagnóstico/pronóstico como es aquél biomarcador que clásicamente identifica pacientes que pueden correlacionarse con un resultado, tales como el cribado de una enfermedad, la monitorización de medicas clínicas y la progresión de la enfermedad, y la predicción del riesgo de enfermedad. Los biomarcadores diagnósticos son firmas que diferencian la población sana de los individuos enfermos o discriminan entre grupos en función de la gravedad de la enfermedad. En las enfermedades raras genéticas, las mutaciones génicas son biomarcadores diagnósticos; (ii) el biomarcador predictivo/terapéutico es aquel que resalta los diferentes resultados de una terapia o tratamiento particular, discriminando entre dos categorías, respondedores y no respondedores, o entre muy y poco respondedores.

\section{Tipos de moléculas}

Entre los tipos de moléculas cabe distinguir los ácidos nucleicos, las proteínas y los metabolitos, medibles en líquidos, células o tejidos orgánicos. Cabe distinguir: (i) biomarcadores genómicos, que incluyen tanto los determinantes de DNA como de RNA, para los que, más allá de la secuenciación Sanger automatizada de la cadena de DNA, se dispone una gran 
versatilidad de técnicas de análisis como son las tecnologías de microarrays, las plataformas de secuenciación masiva (NGS, next generation sequencing), secuenciación de RNA (RNAseq), el análisis de expresión específica de alelos y de isoformas de splicing alternativo, el estudio de la expresión de los microRNA y los estudios de modificación epigenéticos del DNA y del código de histonas; (ii) biomarcadores proteómicos, más cercanos a la función/disfunción biológica y medibles en fluidos orgánicos accesibles (sangre, orina), lo que permite que se puedan monitorizar a lo largo del tiempo y en respuesta a ensayos clínicos. Una de sus limitaciones radica en el hecho de que hay que determinar hasta qué punto reflejan en los fluidos analizados el proceso patológico de los tejidos diana de la enfermedad, lo cual no es óbice para que sea campo de investigación de gran interés. Las técnicas de inmunoensayo y sobre todo las tecnologías de espectrometría de masas han permitido un gran avance en la búsqueda de estos biomarcadores; (iii) biomarcadores metabolómicos: a la par que la proteómica permite encontrar modificaciones de la expresión proteica en un fluido o tejido, el análisis masivo de los metabolitos ofrece también el perfil metabolómico como un biomarcador del estatus de enfermedad y de respuesta a los tratamientos. También aquí la espectrometría de masas y las técnicas de resonancia magnética contribuyen a determinar los perfiles diferenciales entre individuos sanos y enfermos. (iv) biomarcadores de imagen, que determinan estructuras mediante técnicas de resonancia magnética nuclear (RMN) o cambios funcionales/metabólicos empleando trazadores moleculares con es el caso de la tomografía de emisión de positrones (PET) o la tomografía computarizada de emisión monofotónica (SPECT).

El empleo de biomarcadores genómicos nos define el perfil genético del individuo y su susceptibilidad a padecer una enfermedad, actuando como marcador diagnóstico en el caso de las enfermedades raras monogénicas (Boycott, Dyment, Sawyer, Vanstone y Beaulieu, 2014) o como marcador de predicción en el caso de las enfermedades complejas. Por el contrario, los biomarcadores de perfiles bioquímicos (proteómico, metabolómico y tal vez epigenómico) pueden ser útiles para definir el proceso dinámico y evolutivo de la enfermedad y las respuestas a los tratamientos. La combinación del acervo genómico, el perfil bioquímico en fluidos y células, y la estructura tisular/orgánica determinada por técnicas funcionales de imagen hacen del biomarcador un campo de estudio necesario y de primer orden en la medicina personalizada del siglo XXI.

\section{LOS BIOMARCADORES EN LAS ENFERMEDADES RARAS}

Después de lo comentado anteriormente, cabe preguntarse cuál es el papel de los biomarcadores en las enfermedades raras, y especialmente qué tipo de marcadores son relevantes en estas enfermedades que mayoritariamente son genéticas. Desde la perspectiva tanto del diagnóstico, pronóstico y manejo de la historia natural de la enfermedad como del seguimiento y evaluación de la respuesta terapéutica en ensayos clínicos y del tratamiento en la práctica clínica, el papel de los biomarcadores es similar al que tienen en las enfermedades complejas. Tal vez la mayor diferencia entre las enfermedades raras y las comunes radique en el aspecto diagnóstico, dado que, para un número apreciable de enfermedades raras, la mutación génica es un biomarcador de primer orden (Boycott et al. 2014), necesario y generalmente suficiente para el que el individuo desarrolle la enfermedad en algún momento de su biografía. Esto mismo no es posible en las enfermedades comunes complejas, en las que el perfil genómico indica susceptibilidad para desarrollar la enfermedad, esto es, puede ser necesario pero no suficiente (Palau, 2012). No ocurre igual cuando nos interesamos en emplear biomarcadores para el seguimiento de la historia natural o evaluar la respuesta terapéutica. En estas situaciones es tan necesaria la investigación y la detección de nuevos biomarcadores en enfermedades raras como en enfermedades comunes. Si ambos conjuntos de enfermedades comparten mecanismos fisiopatológicos y si, en general, en ambos casos son procesos crónicos, es obvio que conocer y poder determinar en el marco clínico los biomarcadores subrogados que definan el estatus y la evolución del paciente es fundamental para desarrollar nuevas terapias que demuestren su eficacia en los ensayos clínicos, ya sea la enfermedad poco frecuente, ya sea una enfermedad de alta prevalencia.

\section{¿CÓMO ENTENDEMOS, PUES, LA MEDICINA PERSO- NALIZADA EN LAS ENFERMEDADES RARAS?}

Sobre la base de los elementos descritos anteriormente hemos mostrado varios aspectos que pueden diferenciar las enfermedades raras de las comunes. Uno de estos elementos es la edad de inicio de la enfermedad y la afectación del conjunto de la biografía de la persona enferma. Sin embargo, la mayoría de los elementos biológicos y clínicos relacionados con la medicina personalizada y el modelo prospectivo de la enfermedad (Snyderman, 2009: Snyderman y Langheier, 2006; Snyderman y Yoediono, 2006) son compartidos por las enfermedades raras y las enfermeda- 
des comunes. La identificación de perfiles genómicos y biológicos en cada individuo, sea afectado o sano, es relevante para diseñar su cuidado y manejo clínico. ¿Hay, sin embargo, algún aspecto específico de la medicina personalizada en las enfermedades raras? Parte de la respuesta se relaciona con el desarrollo de biomarcadores y lo acabamos de mencionar. Los biomarcadores genómicos son relevantes para la enfermedad común, ya que están definiendo la susceptibilidad de un rasgo o enfermedad; la combinación de las variantes genómicas puede aumentar o disminuir el riesgo de enfermedad en los individuos de una población, de modo que si el perfil genómico de una persona induce una reducción de la línea basal de la enfermedad, esta padecerá la enfermedad más tempranamente en su vida. En las enfermedades raras la línea basal de la persona es muy baja, a raíz de que el individuo es portador de una o dos mutaciones altamente penetrantes cuya presencia es suficiente para que se desarrolle la patología. Los biomarcadores dinámicos bioquímicos son necesarios para definir el pronóstico y la respuesta terapéutica tanto en las enfermedades comunes como en las minoritarias. En ambos casos los biomarcadores deben estar relacionados con las vías biológicas que participan en el proceso de la enfermedad. La diferencia entre las enfermedades comunes y las raras radica pues en el papel central de la mutación primaria patógena en el origen y la evolución ulterior de la enfermedad (Palau, 2012). Conocer esta mutación es fundamental para establecer el manejo del paciente en el marco de la medicina personalizada.

\section{AGRADECIMIENTOS}

Las ideas expuestas tienen su origen en la actividad científica y clínica del autor, con financiación del Ministerio de Ciencia, Innovación y Universidades, del Instituto de Salud Carlos III, del CIBERER, de la Comisión Europea (FP7 y DG SANCO), de la Generalitat Valenciana, de la Generalitat de Catalunya, y de las Fundaciones Ramón Areces, Isabel Gemio y Amigos de Nono.

\section{BIBLIOGRAFÍA}

Berman, J. J. (2014). Rare Diseases and Orphan Drugs. Keys to Understanding and Treating the Common Diseases. Amsterdam: Elsevier Inc.

Bonassi, S. y Neri, M. (2009). Application of biomarkers in human population studies. En: Willard, H. F. y Ginsburg, G. S. (eds.). Genomic and Personalized Medicine. San Diego: Academic Press, pp. 299-307. https://doi.org/10.1016/ B978-0-12-369420-1.00025-1

Boycott, K. M., Dyment, D. A., Sawyer, S. L., Vanstone, M. R. y Beaulieu, C. L. (2014). Identification of genes for childhood heritable diseases. Annual Review of Medicine, 65, pp. 19-31. https://doi.org/10.1146/annurevmed-101712-122108

Ferlini, A., Scotton, C. y Novelli, G. (2013). Biomarkers in rare diseases. Public Health Genomics, 16 (6), pp. 313-321. https://doi.org/10.1159/000355938

Ginsburg, G. S. (2009). Translational genomics: from discovery to clinical practice. En: Willard, H. F. y Ginsburg, G. S. (eds.). Genomic and Personalized Medicine. San Diego: Academic Press, pp. 261-274. https://doi.org/10.1016/ B978-0-12-369420-1.00022-6

Ginsburg, G. S. y Willard, H. F. (2010). The foundations of genomic and personalized medicine. En: Ginsburg, G. S. y Willard, H. F. (eds.). Essentials of Genomic and Personalized Medicine. San Diego: Academic Press / Elsevier Inc., pp. 1-10. https://doi.org/10.1016/B978-012-374934-5.00001-5

Hamburg, M. A. y Collins, F. S. (2010). The path to personalized medicine. New England Journal of Medicine, 363 (4), pp. 301-304. https://doi.org/10.1056/ NEJMp1006304

Palau, F. (2010). Enfermedades raras, un paradigma emergente en la medicina del siglo XXI. Medicina Clínica, 134 (4), pp. 161-168. https://doi.org/10.1016/j. medcli.2009.06.038

Palau, F. (2012). Personalized medicine in rare diseases. Personalized Medicine, 9 (2), pp. 137-141. https://doi. org/10.2217/pme.12.2

Roubertoux, P. L. y Vries, P. J. de (2011). From molecules to behavior: lessons from the study of rare genetic disorders. Behavior Genetics, 41 (3), 341-348. https://doi.org/10.1007/s10519-0119469-y

Scotton, C., Passarelli, C., Neri, M. y Ferlini, A. (2014). Biomarkers in rare neuromuscular diseases. Experimental Cell Research, 325 (1), pp. 44-49. https://doi. org/10.1016/j.yexcr.2013.12.020

Snyderman, R. (2009). The role of genomics in enabling prospective health care. En: Willard, H. F. y Ginsburg, G. S. (eds.). Genomic and Personalized Medicine, San Diego: Academic Press, pp. 378385. https://doi.org/10.1016/B978-012-369420-1.00032-9

Snyderman, R. y Langheier, J. (2006). Prospective health care: the second transformation of medicine. Genome Biology, 7 (2), 104. https://doi.org/10.1186/ gb-2006-7-2-104

Snyderman, R. y Yoediono, Z. (2006). Prospective care: a personalized, preventive approach to medicine. Pharmacogenomics 7 (1), pp. 5-9. https://doi. org/10.2217/14622416.7.1.5 